KIPPING AND POPE: RACEMISM AND PSEUDORACEMISM, 989

\title{
LXXXVIII.--Racemism and Pseudoracemism.
}

By Frederic Stanley Kipping, Ph.D., D.Sc., and William Jackson Pope.

During the course of our work on the $\pi$-derivatives of camphor (Trans., 1893, 63, 547 ; 1895, 67, 354, 371), we have had many opportunities of comparing the physical and crystallographical properties of corresponding optically active and inactive (externally compensated) substances ; the results of this comparison, more especially in some of the cases described in the preceding paper, have led us to conclusions which we desire to place on record.

At the present time, apparently, only two classes of optically inactive solid substances, composed of equal quantities of two enantiomorphous isomerides, are recognised. In the first place, there are mere mixtures, in which each of the two optically active components retains its own characteristic crystalline form, so that it can be separated from its antipodes mechanically if the two forms are distinguishable by the eye; and, in the second place, there are optically inactive externally compensated substances which are not mere mixtures in the above sense, and to which, therefore, the term 'racemic' is at present applied.

It is obvious, however, from a study of the literature of the subject, that this term racemic has no generally accepted definite meaning, and consequently it is sometimes difficult to form an opinion, and opinions will differ, whether a particular substance should or should not be described as a racemic compound. In making this statement, we must not omit to mention that the question as to which properties afford the best criteria of a racemic compound has been recently discussed by Walden (Ber., 1896, 29, 1692), who, from the results of a comparative study of a large number of optically active and externally compensated substances, arrives at a series of conclusions, of which the last runs as follows:- "Als wahre racemische Verbindung kann jede inactiv (aus der d- und l-Form bestehende) Substanz betrachtet werden, sobald sie eine von den activen Componenten verschiedene Krystallform aufweist und eine verschiedene Dichte besitzt, parallel damit kommt ihr gewöhnlich auch ein verschiedener Schmelzpunkt und eine andere Löslichkeit zu."

Now, whether we adopt this conclusion or not, if we examine the properties of some of the inactive substances described in the preceding paper, and then try to group them into two classes, we find that, although some of them may be easily classed as mere mixtures on the one hand, or as racemic compounds on the other, there still remain

vOL. LXXI. 
certain cases in which it is almost impossible to arrive at a satisfactory decision. :

Leaving out of consideration for the present the two $\pi$-chloro- and $\pi$-bromo-camphoric acids and their anhydrides, as many of these compounds, and especially those belonging to the inactive series, do not lend themselves to crystallographic investigation, we will deal in the first place with the active and inactive forms of trans- $\pi$-camphanic acid (preceding paper, p. 973).

The active acid crystallises from ethylic acetate in large, hydrated, monosymmetric prisms, and from cold water in small, less well-defined, hydrated, monosymmetric prisms ; these two forms are crystallographically identical. It also crystallises in another well-defined hydrated form, namely, in long, acicular, orthorhombic prisms; these crystals are obtained when the anhydrous acid is dissolved in anhydrous benzene and the solution allowed to evaporate spontaneously in the air; their melting point is $164-165^{\circ}$.

Anhydrous crystals of the active acid are deposited from its solutions in a mixture of chloroform and light petroleum; these consist of transparent, orthorhombic prisms, which also melt at $164-165^{\circ}$.

Inactive trans- $\pi$-camphanic acid separates from water in small, hydrated, monosymmetric prisms, closely resembling the monosymmetric crystals of the active acid; the two substances are remarkably similar from a crystallographic point of view, the same forms being present on each, and the corresponding angles and axial ratios differing but slightly; nevertheless, the crystals are distinctly different. When, now, the inactive acid is crystallised from ethylic acetate, it is deposited in anhydrous, six-sided, monosymmetric plates, quite different crystallographically from any of the modifications of the active substance. Notwithstanding these marked differences, which may be accepted as conclusive evidence of racemism, this modification of the inactive acid has the same melting point as the active compound.

We shall have to decide, then, whether there are two racemic forms of trans- $\pi$-camphanic acid one of which is hydrated whilst the other is anhydrous, or whether the apparent racemic character of the hydrated form wust be condemned.

Passing on to $c i s-\pi$-camphanic acid (p. 983), we find that here there is no doubt as to the nature of the inactive acid; as already shown, when the inactive acid separates from its solutions, the two optical antipodes of which it is composed are deposited side by side and can be easily identified.

The case of inactive trans-camphotricarboxylic acid also offers no difficulty ; it differs totally from the active acid in crystalline structure, in its crystals being anhydrous, and in other properties, and is therefore a racemic compound. 
Inactive trans-camphotricarboxylic anhydride, however, affords another example of one of those cases in which a decision is much harder to arrive at; it crystallises in monosymmetric prisms, which, like the crystals of the active compound, melt at $253-254^{\circ}$, and the density of the two isomerides is the same within the limits of experimental error (p. 988) ; on the other hand, although the same forms are present on the crystals of both anhydrides, yet the two substances differ appreciably in crystalline form, that is to say, corresponding angles on the two kinds of crystals are only approximately equal.

It will be seen from these examples, and from many similar instances which we quote later, that externally compensated substances which are not mere mixtures, inasmuch as they have a different crystalline form from their components, may be sharply divided into two classes: (a) consisting of inactive compounds the crystallographic properties of which differ in a very marked manner from those of their active components, and $(b)$ consisting of inactive substances the crystallographic properties of which so closely resemble those of their active constituents as to render distinction difficult. The anhydrous crystals of inactive trans- $\pi$-camphanic acid and of inactive trans-camphotricarboxylic acid belong to class (a), whilst the crystals of inactive trans-camphotricarboxylic anhydride and the hydrated crystals of inactive trans- $\pi$-camphanic acid belong to class $(b)$.

Now the term racemic, we take it, should be applied only to those inactive compounds which, like racemic acid, differ from their active components to a very considerable extent crystallographically (and consequently in other properties also), and not to substances such as camphotricarboxylic anhydride and hydrated trans- $\pi$-camphanic acid, which it is difficult to distinguish by crystallographic or other methods from their optically active isomerides. Whilst retaining the name racemic compound for a substance belonging to class $(a)$, we propose, therefore, to call those belonging to class $(b)$ pseudoracemic, in order to distinguish them from mere mixtures of the two antipodes on the one hand, and from racemic compounds on the other.

This proposed classification of externally compensated compounds is not only a convenient distinction to draw between substances which are in fact easily divisible into the three groups, $(a)$ racemic, $(b)$ pseudoracemic, and $(c)$ mere mechanical mixtures, but is, we believe, amply justified by arguments based on a consideration of crystalline structure.

The theory of crystalline structure has, during the last thirty years, been securely founded by the work of Bravais, Sohncke, Fedorow, and more recently by that of Barlow (Zeit. f. Kryst., 1894, 23, 1). It is now known that all crystallographically simple (namely, not twinned or grouped in any way) substances must possess one or other of 230 so-called types of crystalline structure; that is to say, there are 230 
types of homogeneous arrangement of molecular matter in threedimensional space, and every crystallographically simple substance must consist of parts or particles arranged in one or other of these typically different ways. These types differ, firstly, in their general symmetry, and, secondly, in the nature of the repetition in space of their parts or particles; when classified according to their general symmetry, they fall into the 32 holo-, hemi-, and tetarto-hedral crystalline systems, amongst which experience shows that all crystalline substances must be distributed. Now Barlow has shown (Min. Mag., 1896, 11, 119) that the 230 simple homogeneous structures described by him may be symmetrically partitioned into unit groups. When thus partitioned, these structures may, for our present purpose, be divided into $(A)$ those of which the unit groups are identical with their own mirrorimages, or are of two enantiomorphously related kinds in equal numbers, and $(B)$ those of which the unit groups are enantiomorphous and are not thus balanced.

But the chemical molecules of all organic compounds which are optically active in the liquid or amorphous state contain an asymmetric carbon atom, so that such molecules are enantiomorphously related to their mirror-images; since this statement is also true of any aggregate of such molecules, we can consider the unit groups of the partitioned structures as consisting of the chemical molecules. It thus follows that all optically inactive substances possessing homogeneous structures must, when the latter are symmetrically partitioned and dislocated, fall into group $(A)$, whilst the partitioned structures of all optically active compounds must be classified in group $(B)$; in other words, no optically active substance can assume the same type of homogeneous crystalline structure as a racemic compound.

The question now arises as to whether a homogeneous, crystalline, externally compensated, inactive substance can so closely resemble its optically active components in crystalline form as do the active and inactive modifications of trans-camphotricarboxylic anhydride, and of trans- $\pi$-camphanic acid in the hydrated form deposited from aqueous solution. In the face of the above argument, this seems in the highest degree improbable, since, whilst the crystallographic resemblances are so great, and extend not only to the geometrical properties, but also to the orientation of the optical indicatrix and to other properties, the crystalline structures must belong the one to class $(A)$, the other to class $(B)$; and it cannot be believed that these properties, which are almost wholly expressions of the crystalline structure, could be in such close agreement if the latter were so fundamentally dissimilar as the above argument would indicate. Further, if it were possible that crystals having different types of structure should yet exhibit the above similarities, we should expect to find a gradation of the degree 
of similarity between corresponding active and inactive compounds, whereas, as noted above, we actually observe that these isomerides are either extremely dissimilar crystallographically (racemic), or else resemble each other so closely that distinction is difficult (pseudoracemic). We are thus led to the conclusion that the crystals of pseudoracemic substances are not homogeneous, but are merely complicated intercalations of crystals of the right- and left-handed modifications similar in nature to those pseudosymmetrical quartz crystals which are merely interpenetrations of a right- and a lefthanded crystal (Judd, Min. Mag., 1892, 10, 123).

We therefore define a pseudoracemic substance as an intercalation of an equal, or approximately equal, proportion of two enantiomorphously related components, each of which preserves its characteristic type of crystalline structure, but is so intercalated with the other as to form a crystalline individual of non-homogeneous structure. A solid* racemic compound, on the other hand, may be defined as a crystalline substance of homogeneous structure which contains an equal proportion of two enantiomorphously related isomerides.

The relations holding between a mere mixture of optical antipodes, a pseudoracemic substance, and a racemic compound are closely parallel to those existing between a crystalline mixture, an isomorphous mixture, and a double salt. The crystallographic methods, by which a double salt can be distinguished from an isomorphous mixture, may be directly applied to distinguish between racemic and pseudoracemic substances. Thus, according as the crystalline substance obtained from a mixture of two salts resembles or differs from either of its components crystallographically, it is regarded either as an isomorphous mixture or as a double salt; similarly, an inactive externally compensated substance, which closely resembles its active isomerides crystallographically, is to be considered as pseudoracemic, whereas when the contrary is true, it is to be regarded as racemic.

The definitions which we have just given of pseudoracemic and racemic compounds are no doubt based to a great extent upon theoretical considerations, and it might be objected that they would be of little practical value in trying to arrive at a conclusion as to the nature of a given externally compensated substance; we would point out, however, that any such conclusion must be based on a general review of all the properties of such a substance, and that the data thus collected will also amply suffice to show to which of the three classes, $(a)$ racemic, $(b)$ pseudoracemic, and (c) mere mixture, the given inactive externally compensated substance belongs.

In order to make this clear, we must now dicuss the properties of

* The question as to the existence of racemic compounds in the liquid state is not touched upon in this paper. 
racemic and pseudoracemic compounds generally, and draw conclusions which, based chiefly upon the experimental evidence collected during our examination of these compounds, are also supported by facts recorded by others in the literature of the subject.

Pseudoracemic crystals have nearly the same geometrical and optical constants as those of their enantiomorphous components; small, but perfectly distinct, differences between corresponding angles do, however, actually occur, and may be attributed to the disturbing effect of intercalation. The nature of these differences will be evident from the tables already given (pp. 976,988), in which some of the corresponding angles are compared in the cases of active and pseudoracemic trans- $\pi$, camphanic acid and trans-camphotricarboxylic anhydride; other examples are quoted later.

Although crystals of pseudoracemic substances may appear welldefined to the unaided eye, goniometrical examination reveals the fact that their faces show multiple images just as in the case of crystals of impure substances, owing probably in both cases to the disturbance set up by the intercalation of different structures; the crystals of the two compounds just mentioned exhibit this property in a very marked manner. The crystals of racemic compounds, on the other hand, differ so widely from those of their enantiomorphously related components that no more striking similarities than are involved in morphotropic relationships are ever observed either amongst the geometrical or optical properties.

The melting point of a pseudoracemic compound is the same as that of its enantiomorphously related components, provided it does not change its crystalline structure and become a racemic compound below its melting point.

This conclusion seems to be justified by a consideration of the general behaviour of optically inactive externally compensated substances on melting. It is well known that, in a great many cases, an optically inactive substance which, at ordinary temperatures, may be a mere mixture, a pseudoracemic substance or a racemic compound, melts at the same temperature as its active constituents (compare Walden, loc. cit., p. 1696) ; as this result can hardly be fortuitous, it must be assumed that an optically active substance melts at the same temperature whether heated alone or in intimate contact with its optical antipodes, unless the two unite to form a racemic compound. The facts that some compounds which are doubtless racemic at ordinary temperatures melt at the same temperature as their active components, whereas pseudoracemic substances, or mere mixtures of the two antipodes, do not always melt at the same temperature as their active components, can be advanced neither for nor against this view, for the following reasons. Organic compounds are frequently polymorphous, 
and are peculiarly prone to change their type of crystalline structure as the temperature changes; many illustrations of this fact have been given in our previous papers (Trans., 1895, 371). Since the type of crystalline structure assumed by a given substance is dependent on the temperature, and only certain types of crystalline structure are possible for racemic compounds, on the one hand, and for pseudoracemic or optically active substances, on the other (p. 992), it follows that a change in temperature may cause either the resolution of a racemic compound into, or the formation of a racemic compound from, its constituents.

Consequently, a substance which, at ordinary temperatures, is racemic, may melt as such, or it may be first converted into a mixture of the active components, in which case it will melt at the same temperature as the active substances; conversely, a substance which at ordinary temperatures is pseudoracemic or a mere mixture of two optical antipodes, may become racemic when heated, in which case the melting point will be different from that of the active components.

The whole subject of the melting points of such optically inactive substances is, in fact, elucidated by these considerations, and although experimental evidence in their support is somewhat difficult to obtain, we believe that the following will suffice to show that they are well founded.

Racemic $t v^{\circ}$ ans- $\pi$-camphanic acid appears to melt at $164-165^{\circ}$ simultaneously with the active acid, but, before melting, the crystals, which are at first transparent, become quite opaque, owing to a change in crystalline form; if, as we think probable, the result of this crystal line change is the resolution of the racemic compound, it is clear that there is nothing anomalous in the identity of melting point. A microcrystallographic comparison of the crystalline films of the active and inactive acids obtained by melting them on a microscope slide affords very strong evidence that at temperatures just below $164^{\circ}$, the crystalline structure is of the same type, but becomes dissimilar on cooling to ordinary temperatures; the simplest explanation of this is, that the racemic compound, which we have examined crystallographically (p. 977), exists at the ordinary temperature, but is resolved into its components when heated below the melting point.

Very convincing evidence is obtained by the study of trcons-camphotricarboxylic anhydride; we conclude from the geometrical and optical properties, and from the habit and density of the crystals of the inactive substance, that they are pseudoracemic. The active and pseudoracemic anhydrides melt at the same temperature, so that on melting them on a microscope slide and watching the cooling films, both are found to be trimorphous; moreover, the similarity existing between each pair of corresponding crystalline modifications is so great that the two 
forms are indistinguishable; in this case, we must conclude that no racemic compound is formed on heating the pseudoracemic substance, and the melting points of the active and inactive anhydrides are consequently identical.

Inactive $\pi$-bromocamphor (Trans., 1895, 67, 387), as deposited from solution, is doubtless racemic, but its melting point (about $92 \cdot 7^{\circ}$ ) is so close to that of the corresponding active compound $\left(93 \cdot 4^{\circ}\right)$ that a difference is not easily established, especially as the melting point of the inactive substance is not quite sharp. The examination of crystalline films of the two substances shows that, on cooling, they both yield at first a cubic modification, but whereas the inactive substance only changes once (doubtless to the racemic compound), the active isomeride first undergoes conversion into one, and then into a second doubly refracting modification. Further, mixtures of active and racemic $\pi$-bromocamphor melt at practically the same temperature as the separate constituents. This behaviour seems to indicate that the racemic compound becomes a mere mixture when heated, and has conseguently the same melting point as the active substance; we are not disposed to attribute much importance to the very trivial difference between the observed melting points of the active and inactive substances, because it is quite possible that this difference may be due to the change in crystalline form of the latter taking place very near the melting point.

The inactive and active forms of $\pi$-chlorocamphor show a behaviour which is, on the whole, analogous to that of the corresponding bromoderivatives, and leads, therefore, to the same conclusions.

Inactive $c i s-\pi$-camphanic acid does not form a racemic compound when deposited from solution at ordinary temperatures, and melts at the same temperature as the optically active substance; a mixture of the optically active and inactive acids has also the same melting point as the former.

Active $\pi$-bromocamphoric anhydride, the inactive substance (which seems to be pseudoracemic), and a mixture of the two, all melt at the same temperature ; trans-camphotricarboxylic anhydride shows a similar behaviour.

The above cases, all of which have arisen during our study of the $\pi$-derivatives of camphor, are quoted as examples either of the resolution of racemic compounds or of the non-formation of racemic from pseudoracemic substances or mixtures on heating; as examples of the converse kind, namely, of the formation of racemic compounds from mere mixtures or pseudoracemic substances on heating, we instance the following.

Dextro- and lævo-camphorsulphonic chlorides crystallise in large, orthorhombic tetrahedra (Trans., $1893,63,565$ ), which melt at $137 \cdot 5^{\circ}$; 
the inactive substance crystallises in large prisms, having apparently the same axial ratios as, and similar optical properties to, those of the crystals of the active compounds, but which, as we previously pointed out (loc. cit., p. 561), seem to be produced by twinning, as in the case of quartz. These crystals, which bear every mark of being pseudoracemic, melt at about $106^{\circ}$, namely, $30^{\circ}$ lower than those of the active compounds. The behaviour of the camphorsulphonic bromides is closely analogous to that of the chlorides (Trans., 1895, 67, 359); the active compound melts at $144-145^{\circ}$, whilst the pseudoracemic substance melts at $115-120^{\circ}$, doubtless owing to the formation of a racemic compound.

An intimate mixture of the enantiomorphously related methylmannosides (E. Fischer and Beensch, Ber., 1896, 29, 2927) melts at $165-166^{\circ}$, namely, at the same temperature as the racemic compound ; the active compounds melt separately at $190-191^{\circ}$. As inactive methylmannoside is known to form a racemic compound when deposited from solution at or above a certain temperature, whilst it gives a mere mechanical mixture of the two antipodes at lower temperatures, there is here very convincing evidence that the depression of the melting point is due to the formation of a racemic compound.

The case of the gulonic lactones, also described by Fischer (Ber., $1892,25,1027)$, is very similar to the foregoing. The two inactive isomerides melt at $181^{\circ}$, and do not yield a racemic compound when deposited from solution at the ordinary temperature; an intimate mixture of the two components, however, melts at $160^{\circ}$. Fischer himself remarks: "vielleicht entsteht unter diesen Bedingungen die wirkliche racemische Verbindung," and doubtless this is so.

A large number of similar cases might be quoted, but the above amply suffice for our purpose. We conclude, therefore, that if an externally compensated substance melts as a mixture or whilst pseudoracemic, its melting point is that of its active components, but if it melts whilst racemic, its melting point is not the same as that of the active isomerides; further, it may be expected that if the substance melts as a racemic compound, the melting point would be depressed in presence of a slight excess of one or other enantiomorphous component, but that no depression would occur under similar conditions if the substance melted as a mere mixture or whilst pseudoracemic.

It follows immediately that the melting point affords no conclusive evidence as to whether a given inactive substance is or is not a racemic compound when crystallised at the ordinary temperature. It is no doubt true that when an inactive substance melts at a higher temperature than its components we are justified in regarding this fact as indicative of the existence of a racemic compound, as already suggested by E. Fischer (Ber., 1894, 27, 3225), but it cannot be accepted as a proof of the same. Fischer's argument (loc. cit.), that, because 
mixtures of two substances generally melt at a lower temperature than either constituent, therefore it is impossible to draw conclusions as to the racemic character of an externally compensated substance which has a lower melting point than its components, is far less convincing; the fact that, in such a case, the melting point is depressed is, in our opinion, due to the formation or existence of a racemic compound at the melting point, but again there is no evidence as to whether the latter exists at ordinary temperatures.

Much, however, may be learned by the method of examination which we have adopted in many cases, namely, by studying the changes undergone during cooling by very thin films of the externally compensated substance. Lehmann has shown at length (Molekularphysik, 1888, $1,119)$ that such microcrystallographic methods are of great service, and we ourselves have found, on several occasions, that important information may often be obtained in this way (compare Trans., 1895, 67,387 , and preceding paper, p. 982 ).

The solubility of a pseudoracemic substance, like that of a racemic compound, is different from that of the enantiomorphous components taken separately.

Much misconception appears to prevail with regard to this question, for, although it seems to be generally understood that the solubility of a racemic compound is not the same as that of either of its active components (compare Wallach, Annalen, 1895, 286, 137), conflicting views are held as to the solubility of a mere mixture of two optical antipodes. Walden, for example, states that the solubility of such a mixture should be twice that of its enantiomorphous constituents (loc. cit., p. 1704). There would seem, however, to be no experimental or theoretical justification for this statement, or for supposing the existence of any simple numerical relation between the solubility of a mechanical mixture of two enantiomorphs or of a racemic compound on the one hand, and that of one of the enantiomorphs on the other. That no simple relation holds in the case of a racemic compound, is shown by numerous examples (compare Walden, loc. cit.), and that the same is true in the case of mere mixtures of two antipodes is proved by Scacchi's determinations of the ratio of the solubilities of active sodium ammonium tartrate and of a mixture of the corresponding dextro- and lævorotatory compounds (kiend. soc. reale di Napoli, 1864, 4, 250). This ratio was found to be $1: 1.412$ at $12.8^{\circ}$ and $1: 1.271$ at $26.2^{\circ}$, the temperature in both cases being below that at which the racemic salt separates from solution.

According to Ladenburg (Ber., 1894, 27, 3065; 1895, 28, 164), the fact that a mixture of two optical antipodes containing excess of one or other modification can be separated by crystallisation into fractions having different specific rotations, is evidence of the presence of a 
racemic compound in the mixture. The case of camphorsulphonic chloride, however, seems to disprove this view; as we have previously shown (Trans., 1893, 63,547), the dextrorotatory substance can be easily isolated from a mixture of the two antipodes when it is present in excess, but, at the same time, the inactive substance cannot be regarded as a racemic compound. Camphorsulphonic bromide shows exactly the same behaviour (Trans., 1895, 67, 354).

It would seem, therefore, that solubility determinations are useless at present as a means of proving whether a particular crystalline substance is racemic, pseudoracemic, or a mere mixture, and conclusions which, like those of Aschan (Actc Soc. Scient. fenn, 21, [v], $206)$ in the case of $w$-bromocamphoric anhydride, are based mainly on the solubility, have consequently little weight.

It is, indeed, doubtful whether the enormous difference in solubility of the active and inactive calcium galactates (Fischer, Ber., 1894, 27, 3225 ) can be accepted as conclusive evidence of the racemic nature of the latter.

It would be expected that a valuable criterion of pseudoracemism might be found in the density; since a pseudoracemic substance is merely an intercalation of two crystalline structures of equal density, there is no obvious reason for assuming that any change will occur. In this connection, however, it has still to be ascertained whether the disturbances in growth attending intercalation are of sufficient magnitude to appreciably affect the density; in the only case which we have been able to examine satisfactorily, namely, that of trans-camphotricarboxylic anhydride, the active and inactive substances were found to have the same density within the limits of experimental error. Cases such as those of dipentene tetrabromide (Hintze, Annalen, 1885, 227, 278) and of inactive carvoxime (P. Beyer, Zeits. f. Kryst., 1890, 18, 296) are, however, on record in which the crystalline form of the active and inactive compounds are remarkably similar, but in which the densities are appreciably different (Liebisch, Annalen, 1895, 286, 140) ; the crystals of dipentene tetrabromide exhibit the characteristics of pseudoracemic compounds with particular distinctness. Further investigation of these compounds is obviously desirable.

Racemic compounds apparently conform to Liebisch's rule (Annalen, $1895,286,140)$, which states that their crystals have a higher density than those of their optically active components.

Since, in the foregoing pages, we have dealt almost exclusively with facts relating to compounds which we ourselves have studied, it is now necessary to refer briefly to other inactive substances and to examine the data which have been interpreted by others as proofs of racemism; from this examination, we hope to show that pseudoracemism is of common occurrence. 


\section{KIPPING AND POPE: RACEMISM AND PSEUDORACEMISM.}

Dextrorotatory and inactive $w$-bromocamphoric anhydrides, both of which melt at $216^{\circ}$, have been prepared by Aschan (loc. cit., p. 193), who concludes that the inactive substance is racemic because its solubility in chloroform is different from that of the active compound; that this cannot be accepted as evidence, is shown above. Further, Aschan's two preparations were examined crystallographically by Wiik (loc. cit., pp. 203, 205); they are both orthorhombic and the axial ratios are but slightly different, being

$$
a: b: c=0.8880: 1: 0.5775
$$

in the case of the dextrorotatory substance, and

$$
a: b: c=0.8725: 1: 0.5799
$$

in the case of the inactive compound. Wiik also states that the crystals of the inactive anhydride are smaller and less well-developed than those of the active substance ; that they show vicinal faces and can be measured only approximately. All these facts point to the conclusion that inactive $w$-bromocamphoric anhydride is a pseudoracemic substance.

Active carvone tribromide melts at $88-89^{\circ}$ and forms rhombic crystals (Liebisch, Annalen, 286, 142) which show the principal angles

$$
110: 1 \overline{1} 0=50^{\circ} 37^{\prime} ; 011: 0 \overline{1} 1=76^{\circ} 53 \frac{1}{2}^{\prime} ; 110: 011=74^{\circ} 42 \frac{1}{2}^{\circ} \text {. }
$$

The inactive substance melts at $65^{\circ}$ and is also rhombic, showing the corresponding angles

$$
110: 1 \overline{1} 0=50^{\circ} 31^{\prime} ; 011: 0 \overline{1} 1=76^{\circ} 52^{\prime} ; 110: 011=74^{\circ} 29^{\prime},
$$

but it would seem from the incomplete measurements that these crystals were less well-developed than those of the active tribromide; the density of the two substances is the same. Here again, therefore, it would seem that the inactive substance is either pseudoracemic or a mere mixture, but that it becomes racemic when heated.

Dipentene hydrochloride nitrolanilide seems to be merely a pseudoracemic intercalation of the oppositely active limonene compounds; P. Beyer (Zeits. f. Kryst., 18, 296) found the crystals of the active and inactive substances to be anorthic, and gives the following axial data respectively :- $a: b: c=0.71036: 1: 1.0351 . \quad a=101^{\circ} 9^{\prime} ; \beta=103^{\circ} 4^{\prime}$; $\gamma=78^{\circ} 22^{\prime}$, and $a: b: c=0.70613: 1: 0.98191, \alpha=101^{\circ} 6^{\prime} ; \beta=102^{\circ} 39^{\prime}$; $\gamma=78^{\circ} 18^{\prime}$. The only appreciable differences between the two kinds of crystals occur in the zone [010], and Beyer notes that in the crystals of the dipentene compound the angles in this zone are not constant, and much striation is observed. The melting points of the active and inactive substances differ considerably, owing, doubtless, to racemation occurring before melting. 
Inactive phenyl- $\alpha \beta$-dibromopropionic acid would also seem, from Fock's description (Ber., 1893, 26, 832), to be pseudoracemic.

The data given for many other compounds, such as urimidosuccinic acid (Grattarola, Atti soc. Tosca. sc. nat., 1890, 11, 119), phellandrene nitrite (Wallach, Annalen, 1888, 246, 235), and others mentioned by Walden (loc. oit., pp. 1696 and 1706), do not afford satisfactory proofs of racemisn or of pseudoracemism, as it is not shown that each crystal really contains the two enantiomorphous isomerides. The case of dextro- and lævo- $\beta$-asparagine is, however, an especially instructive one ; La Valle (Gazzetta, 1887, 17, 226) states that the inactive substance consists of right- and left-handed hemihedral crystals, and frequently the two antipodes are twinned in parallel positions, so that if these contact trins became completely interpenetrant, they would result in an apparently holohedral individual. The latter is exactly the kind of association which seems to occur in the formation of a pseudoracemic crystal.

Chemical Drpartament,

Central Technical College,

City and Guilds of London Institute. 